\title{
Fluorescence in the Active Galactic Nuclei NGC 4151
}

\author{
Mattias Eriksson ${ }^{1}$ and Hans Veenhuizen ${ }^{2}$ \\ ${ }^{1}$ Blekinge Institute of Technology, \\ SE-371 79 Karlskrona, Sweden \\ email: meo@bth.se \\ ${ }^{2}$ Linnaeus University, School of Computer Science, Physics and Mathematics \\ 39182 KALMAR, Sweden \\ email: hans.veenhuizen@lnu.se
}

\begin{abstract}
The possibility of UV lines from Fe II formed in the BLR of NGC 4151 has been examined. As a result Fe II emission has been shown to play an important role for the topology of the 2000 to $3000 \AA$ wavelength region of the NGC 4151 spectra. The Fe II UV emission originates from two processes, collisional excitation and PAR (photoexcitation by accidental resonance).
\end{abstract}

Keywords. galaxies: active, galaxies: nuclei

\section{The model spectra}

NGC 4151 has regions in which emission lines with widths corresponding to several thousand $\mathrm{km} / \mathrm{s}$ doppler broadening are formed. The $\mathrm{Mg}$ II resonance doublet is one of those broad lines (Leech et al. 1987). Since the Fe II $\left({ }^{5} \mathrm{D}\right) 4 \mathrm{p}$ levels have similar ionization and excitation potentials as the Mg II $3 p$ levels, broad emission lines are likely formed by Fe II too. However, in the case of Fe II the flux would be split in more than hundred individual emission lines which would be difficult to detect. The topology of the resulting emission from all those Fe II emission lines is modeled using the oscillator strengths of Kurucz (1988) and Fuhr et al. (1988). In addition to the Fe II collisionally excited lines there is a possibility for PAR excited Fe II lines to be formed in NGC 4151. When $\mathrm{Fe}^{+}$ ions are subjected to strong emission from $\mathrm{H}$ Ly $\alpha$ and $\mathrm{C}$ IV $\lambda 1550$ the Fe II levels $\mathrm{y}^{4} \mathrm{H}_{11 / 2}$ and $\left({ }^{3} \mathrm{~F}\right) 4 \mathrm{p}^{4} \mathrm{G}_{9 / 2}$ levels can be pumped resulting in a number of fluorescence lines in the 2000 to $3000 \AA$ region. This phenomenon has previously been confirmed in symbiotic stars (Johansson 1983). Since the H Ly $\alpha$ and C IV $\lambda 1550$ lines are the strongest UV lines in the NGC 4151 spectra PAR is incorporated in the model spectra.

\section{Results}

The model spectra and observed archive STIS spectra were compared in the 2000 - $3000 \AA$ region. The fractional average difference between the best-fitting model and observations was $\sim 0.08$ when excluding the Fe II emission. When incorporating the Fe II emission the fractional average difference decreased to $\sim 0.03$.

\section{References}

Fuhr, J. R., Martin, G. A., \& Wiese, W. L. 1988, J. Phys. Chem. Ref. Data, 17, Suppl. 4 Johansson, S. 1983, MNRAS, 205, 71

Kurucz, R. L. 1988, Astrophysics \& Space Science, 138, 41

Leech, K. J., Penston, M. V., Snijders, M. A. J., \& Gull, T. R. 1987, MNRAS, 225, 837 